Research Paper

\title{
Ubiquitin specific peptidase 5 enhances STAT3 signaling and promotes migration and invasion in Pancreatic Cancer
}

\author{
Jie Lian ${ }^{1,2^{*}}$, Chao Liu ${ }^{1,2^{*}}$, Xin Guan ${ }^{1,2}$, Bojun Wang ${ }^{1,2}$, Yuanfei Yao ${ }^{1,2}$, Dan Su ${ }^{1}$, Yue ma ${ }^{1}$, Lin Fang ${ }^{1,2}$, Yanqiao \\ Zhang ${ }^{1,2 \llbracket}$ \\ 1. Department of Gastrointestinal Medical Oncology, Harbin Medical University Cancer Hospital, Harbin, Heilongjiang Province, China. \\ 2. Translational Medicine Research and Cooperation Center of Northern China, Heilongjiang Academy of Medical Sciences, Harbin, Heilongjiang Province, China. \\ *These authors contributed equally to this work. \\ $\triangle$ Corresponding author: Yanqiao Zhang, E-mail: yanqiaozhang@ems.hrbmu.edu.cn. \\ (c) The author(s). This is an open access article distributed under the terms of the Creative Commons Attribution License (https://creativecommons.org/licenses/by/4.0/). \\ See http://ivyspring.com/terms for full terms and conditions.
}

Received: 2020.05.22; Accepted: 2020.09.14; Published: 2020.09.30

\begin{abstract}
Purpose: Ubiquitin specific peptidase 5 (USP5) has been reported to promote the progression of several malignant tumors. It may affect cancer development via modulating cell cycle and colony formation. In pancreatic cancer, the biological function of USP5, especially in migration and invasion remains unclear.

Methods: USP5 protein expression levels in primary pancreatic cancer and lymph node metastasis tissues were detected using immunohistochemistry $(\mathrm{IHC}) \cdot \chi^{2}$ test, Kaplan-Meier analysis, univariate and multivariate analyses were used to evaluate the relationship between USP5 expression and clinicopathological feature. RT-qPCR were carried out to quantitate the mRNA expression levels of USP5 in pancreatic cancer cell lines. CCK8 and Colony formation assay were performed to prove how USP5 works in proliferation. Evaluation of tumor metastasis was made by Transwell and wound healing assay. EMT and STAT3 signaling related markers were detected by western blot.

Results: (1) USP5 protein expression levels were related to tumor differentiation, CEA and CA19-9 level. (2) Univariate and multivariate analyses showed that high USP5 expression is an unfavorable prognostic factor for pancreatic cancer. Kaplan-Meier analysis directly indicated that patients with high USP5 expression had shorter overall survival. (3) Increased USP5 expression is related to pancreatic cancer in both proliferation and metastasis. (4) USP5 was proved to mediate STAT3 signaling in pancreatic cancer cells.

Conclusions: The results suggest that USP5 is highly expressed and might have clinical significance for pancreatic cancer patients. High USP5 expression promotes both progression and metastasis by activating STAT3 signaling. Thus, USP5 might be a potential target in pancreatic cancer treatment.
\end{abstract}

Key words: USP5, proliferation, metastasis, pancreatic cancer, deubiquitination, STAT3 signaling

\section{Introduction}

Pancreatic cancer is an aggressive tumor with higher malignancy and higher mortality rates. Accounting for, $80 \%$ of all cases were found distant metastasis at the time of diagnosis [1, 2]. Therefore, it will be meaningful to screen biomarkers and find targeted therapies for pancreatic cancer.

Ubiquitination is a major mechanism in post-translational modification of protein. Deubiquitinating enzymes (DUBs) regulate protein degradation by deubiquitinating the substrate protein [3]. Accumulating evidence has confirmed that ubiquitin-specific proteases (USPs), as an important family of DUBs, have been involved in tumorigenesis and malignancy by regulating other proteins. USP5 is one of the important members of USP family. USP5 have been reported as an oncoprotein in diverse cancers including myeloma [4], non-small cell lung cancer [5], hepatocellular carcinoma [6], colorectal cancer [7], ovarian cancer [8] and pancreatic cancer [9]. USP5 have been studied in the development of pancreatic cancer $[9,10]$. However, the role of USP5 in pancreatic cancer still needs to be studied.

In this study, we reported that USP5 is highly expressed in pancreatic cancer, especially in 
metastasis. The expression of USP5 is associated with poor prognosis. We also found that increased USP5 expression is related to pancreatic cancer in both proliferation and metastasis in vitro. USP5 was also proved to mediate STAT3 signaling in pancreatic cancer cells. This study indicated that USP5 might be a potential target for the treatment of pancreatic cancer.

\section{Materials and Methods}

\section{Patients and samples}

Inclusion criteria of patients was described as follows, (1) Patients underwent surgery and following treatment at Harbin Medical University Cancer Hospital from December 2003 to June 2010 with pathologically confirmed pancreatic cancer. (2) All patients signed informed consent. (3) Patients with adequate pathological tissue. Among them, 11 patients contained primary tumor tissues and its corresponding lymph node metastasis tissue samples. We excluded patients who did not sign the informed consent or with no enough tumor tissue samples. The samples were stored at $-80{ }^{\circ} \mathrm{C}$ before paraffinembedded. This study was approved by the Ethics Committee of Harbin Medical University Cancer Hospital. The clinical pathological features of patients were extracted from medical records.

\section{Immunohistochemical staining (IHC)}

The expression of USP5 in pancreatic cancer tissues was detected using IHC. The sections were processed for immunohistochemical staining as previously reported [11]. The 4 - $\mu$ m-thick sections were deparaffinized before heated in citrate buffer $(0.01 \mathrm{M})$. Then they were incubated with $0.3 \% \mathrm{H} 2 \mathrm{O} 2$ and rehydrated. After blocking, the sections were incubated with anti-USP5 (1:100 Proteintech Wuhan Sanying, Wuhan, China) antibody at $4{ }^{\circ} \mathrm{C}$ overnight. After washing several times in phosphate buffered saline (PBS), the sections were incubated in the biotinylated secondary antibodies. After that, the slides were washed in PBS again, exposed to diaminobenzidine, and counterstained with hematoxylin. After serial dehydration, the slides were mounted under a coverslip for microscopic examination. As for blank control and negative control, the slides were incubated with PBS and omitting the primary antibody, respectively. The intensity of USP5 staining was scored as 0 (no signal), 1 (weak), 2 (moderate), and 3 (marked). Percentage score was assigned as 1 (0-25\%); 2 (26-50\%); 3 (51$75 \%)$; and 4 (76-100\%). The scores of each tumor sample were multiplied to give a final score of 0-14. Two pathologists were asked to evaluate and record the IHC results in all cases independently, without prior knowledge of the clinical data. With a critical value of 9 , we divided the expression to low expression $(<9)$ and positive expression $(\geq 9)$.

\section{Cell culture and transfection}

Human pancreatic cancer cell lines (PL45, PSN1, MIAPaCa2, Panc1, HPAFII, panc03.27, BxPC3, Capan2, AsPC1, CFPAC1, Hs766T, SW1990, Capan1) and the normal pancreatic ductal epithelial cells (HPDE6.C7) were purchased from American Type Culture Collection. All cell lines have been authenticated recently. Cells were maintained in RPMI 1640 (HPDE6.C7 AsPC1, Panc03.27, PSN1, HPAFII), DMEM (SW1990, BxPC3, Panc1, PL45, Hs766T, MiaPaCa-2), IMDM (CFPAC1, Capan1), McCoy's 5A (Capan2) medium. All the cell lines were supplemented with 10\% fetal bovine serum (FBS) and $1 \% \mathrm{PS}$, except for MiaPaCa-2 (10\%FBS and $2.5 \%$ horse serum). They were kept at $37^{\circ} \mathrm{C}$ with $5 \% \mathrm{CO} 2$ in an incubator.

We selected Panc1 cells with low USP5 expression for USP5 gene overexpression and AsPC1 cells with high USP5 expression for USP5 gene knockdown. Both cell lines were seeded in 6-well plates at a concentration of $3 \times 10^{5}$ cells/well. Both overexpression and shRNA plasmid (Vigene Biosciences, Shandong, China) was transfected using Lipofectamine 2000 transfection reagent (Thermo Fisher Scientific, USA). Empty pENTER plasmid and shRNA control were used as Negative control.

\section{Quantitative real-time PCR (RT-qpcr) assay}

Total RNA was extracted by using the Trizol reagent (Thermo fisher Scientific, Waltham, MA, USA), and the reverse-transcription reactions were performed by using an M-MLV Reverse Transcriptase kit (Invitrogen, Carlsbad, CA, USA). To find out mRNA levels of USP5 and $\beta$-actin, RT-qPCR was performed by using a standard SYBR Green PCR kit (Toyobo Life Science, Shanghai, China), according to the manufacturer's instructions. The primers were as follows:

- USP5-F: GCTGCTGTCAGTATTACCGAC, and;

- USP5-R: AAAGCCCAGAAACGTGTTCATA.

\section{Cell viability assay}

Panc1 and AsPC1 cells were treated with siRNAs and overexpression plasmids. The next day, cells were seeded at a density of 1000 cells per well in 96 well plates. Forty-eight hours later, $10 \mu \mathrm{L}$ of Cell Counting Kit-8 reagent (Dojindo, Kumamoto, Japan) was added into each well, and cultured for 1 hour at $37^{\circ} \mathrm{C}$ in the dark, according to the manufacturer's instruction. The absorbance of each well was measured at $450 \mathrm{~nm}$ 
every day for the next 3 days.

\section{Colony formation assay}

Panc1 cells (1000 cells/well) and AsPC1 cells (1000 cells/well) were seeded into 6-well plates and cultured for 7 days, respectively. When the clone is visible, the plate was stained with crystal violet solution for $3 \mathrm{~min}$ and then washed with $\mathrm{ddH}_{2} \mathrm{O}$.

\section{Invasion assay}

As for invasion assays, Panc1 cells $\left(1 \times 10^{5}\right.$ cells/well) and AsPC1 cells $\left(1 \times 10^{5}\right.$ cells/well $)$ were added into top chamber of Transwell inserts coated with $40 \mu \mathrm{L}$ Matrigel (1:15,Corning, NY, USA). The bottom chamber was filled with $750 \mu \mathrm{L}$ of medium containing with $10 \%$ FBS, while the top chamber was filled with $500 \mu \mathrm{L}$ of pure medium. After cultured at $37^{\circ} \mathrm{C}$ for 24 hours, the chamber was washed in PBS and fixed in $4 \%$ paraformaldehyde for twenty minutes. After that, it was stained with crystal violet staining solution (Beyotime, Shanghai, China) and taken pictures under a microscope. The counting was analyzed and quantified via ImageJ (National Institutes of Health).

\section{Migration assay}

Panc1 cells $\left(4 \times 10^{4}\right.$ cells/well $)$ and AsPC1 cells $\left(3 \times 10^{4}\right.$ cells/well $)$ with different treatments were seeded into scratch chamber. The chamber was moved the next day and taken pictures under the microscope (0 h, $24 \mathrm{~h}$ ).

Transwell inserts without Matrigel was used as migration assay.

\section{Western blotting}

Total proteins were harvested from cultured cells as described previously [11]. Proteins were separated by $10 \%$ SDS-polyacrylamide gel electrophoresis and then transferred to PVDF membranes. $5 \%$ nonfat milk was used to block the membrane. After that, the membrane was incubated with primary antibodies against USP5 (1:1000 Proteintech Wuhan Sanying, Wuhan, China), actin (1:5000, Santa Cruz Biotechnology, lnc., Dallas, TX, U.S.A.), STAT3 (1:1000, Affinity, Changzhou, China), pSTAT3 (1:500, Affinity, Changzhou, China) and other EMT markers (N-cadherin, 1:1000, Proteintech Wuhan Sanying, Wuhan, China, vimentin, 1:1000, Arigo, Taiwan, China, $\beta$-catenin, 1:1000, Arigo, Taiwan, China), followed by horseradish peroxidase (HRP)conjugated secondary antibodies (1:5000 Proteintech Wuhan Sanying, Wuhan, China). Immunoreactive proteins were detected using a chemiluminescence solution (Thermo fisher Scientific).

\section{Bioinformatics analysis}

GEPIA and ICGC databases were used to validate our experimental results. GEPIA directly showed the differential expression of USP5 in pancreatic cancer and normal tissues. As for ICGC, we downloaded and re-analyzed USP5 expression and its correlation with clinical prognosis.

\section{Statistical analysis}

All the results were presented as the mean \pm standard using GraphPad prism 7.0 (GraphPad Software, USA) and SPSS statistical software 18.0 (IBM Corp., Armonk, NY, USA). $\mathrm{X}^{2}$ test was used to analyze the relationship between USP5 expression and other clinicopathological features. The Cox proportional hazard model was generated for univariate and multivariate regression analysis to identify prognostic factors. Kaplan-Meier survival curves were constructed using a logrank test. Pearson correlation coefficient analysis was conducted to test for correlation between two quantitative measurements. One-way analysis of variance (ANOVA) and Tukey's posttest were used to compare multiple groups of data. $P$-value $<0.05$ was considered statistically significant; for all results * denotes $P<0.05 ;{ }^{* *} P<0.01$ and ${ }^{* * *} P<0.001$. Each experiment was performed with at least three independent experiments.

\section{Results}

\section{Association between USP5 and the clinico- pathological features in pancreatic cancer patients}

To identify the regulatory roles of USP5, USP5 protein levels were tested in 94 pancreatic cancer patients by IHC. Among the samples, 61 patients had high expression of USP5 in tumor tissues (details shown in Table 1). The results showed that USP5 expression level is related to tumor differentiation, CEA and CA19-9 levels $(P<0.05)$.

\section{High USP5 expression level is an independent prognostic factor in pancreatic cancer}

As shown in Table 2, univariate analysis found that USP5 expression $(P=0.001)$, tumor differentiation $(P<0.001)$, CEA level $(P<0.05)$ and CA19-9 level $(P<$ $0.01)$ were significant prognostic predictors in pancreatic cancer. Multivariate analysis identified that USP5 expression $(P<0.05)$ and differentiation $(P<$ 0.05 ) were independent prognostic factors. KaplanMeier survival analysis was performed to explore the association between USP5 expression and survival time in pancreatic cancer patients (Fig. 1A). We found that the median OS of patients with positive USP5 
expression (12.9 months) was remarkably shorter than patients with negative USP5 expression (22.8 months). In general, the results indicated that patients with negative USP5 expression have a longer survival time than those with positive USP5 expression in pancreatic cancer. In the public GEPIA database, we also found that USP5 expression was significantly higher in pancreatic cancer tissue than in normal tissue (Fig. 1B). Additionally, we verified KaplanMeier survival analysis in ICGC Data Portal (ICGCPACA-AU), which is consistent with our result (Fig. 1C).

Table 1. Correlation between USP5 expression and clinicopathological features of patients with pancreatic cancer

\begin{tabular}{|c|c|c|c|}
\hline \multirow[t]{2}{*}{ Clinicopathological feature } & \multicolumn{2}{|c|}{ USP5 expression } & \multirow[t]{2}{*}{$P$ value } \\
\hline & Low & High & \\
\hline Age (years) & & & 0.8877 \\
\hline$\leq 50$ & 8 & 14 & \\
\hline$>50$ & 25 & 47 & \\
\hline Sex & & & 0.3789 \\
\hline Male & 22 & 35 & \\
\hline Female & 11 & 26 & \\
\hline Tumor site & & & 0.6346 \\
\hline Pancreatic head & 19 & 32 & \\
\hline Pancreatic body and tail & 14 & 29 & \\
\hline Differentiation & & & 0.02359 \\
\hline High differentiation & 10 & 7 & \\
\hline Middle and low differentiation & 23 & 54 & \\
\hline T stage & & & 0.3868 \\
\hline $\mathrm{T} 1.2$ & 9 & 22 & \\
\hline T3.4 & 24 & 39 & \\
\hline N stage & & & 0.9941 \\
\hline N0 & 30 & 54 & \\
\hline N1 & 3 & 7 & \\
\hline M stage & & & 1.0000 \\
\hline M0 & 32 & 59 & \\
\hline M1 & 1 & 2 & \\
\hline AJCC (pTNM) & & & 0.9508 \\
\hline I & 9 & 17 & \\
\hline IIIIIIV & 24 & 44 & \\
\hline CEA & & & 0.0324 \\
\hline Normal & 28 & 39 & \\
\hline Abnormal & 5 & 22 & \\
\hline CA199 & & & 0.0133 \\
\hline Normal & 13 & 10 & \\
\hline Abnormal & 20 & 51 & \\
\hline
\end{tabular}

Table 2. Univariate and multivariate analysis of clinicopathological factors in patients with pancreatic cancer

\begin{tabular}{lllll}
\hline Variable & \multicolumn{3}{c}{ Univariate analysis } & \multicolumn{3}{c}{ Multivariate analysis } \\
\cline { 2 - 5 } & HR $(95 \% \mathrm{CI})$ & $P$ value & HR $(95 \% \mathrm{CI})$ & $P$ value \\
\hline Sex & $0.71(0.449-1.133)$ & 0.152 & & \\
Age & $1.61(0.928-2.805)$ & 0.898 & & $\mathbf{0 . 0 4 4}$ \\
USP5 & $2.28(1.392-3.734)$ & $\mathbf{0 . 0 0 1}$ & $1.72(1.014-2.90)$ & \\
Tumor site & $0.69(0.440-1.089)$ & 0.112 & & $\mathbf{0 . 0 1 4}$ \\
Differentiation & $0.29(0.148-0.561)$ & $<\mathbf{0 . 0 0 1}$ & $2.44(1.200-4.940)$ & \\
T stage & $1.12(0.708-1.773)$ & 0.627 & & \\
N stage & $1.11(0.554-2.229)$ & 0.765 & & \\
M stage & $2.42(0.750-7.799)$ & 0.139 & & 0.729 \\
pTNM & $0.94(0.580-1.509)$ & 0.784 & & 0.084 \\
CEA & $060(0.373-0.952)$ & $\mathbf{0 . 0 3 0}$ & $0.91(0.543-1.534)$ \\
CA199 & $0.43(0.248-0.753)$ & $\mathbf{0 . 0 0 3}$ & $0.58(0.315-1.075)$ & \\
\hline
\end{tabular}

Increased USP5 expression suggests correlation with pancreatic cancer metastasis

We detected USP5 expression in 11 pairs of primary pancreatic tumors and lymph node metastasis tissues (Fig. 2A). Interestingly, the results showed that the expression of USP5 was elevated in patients with lymph node metastasis (Fig. 2B). The USP5 mRNA level was detected in normal pancreatic cell and multiple pancreatic cancer cells, showing that USP5 mRNA expression was significantly increased in metastasis cells than primary cancer cells (Fig. 2C). We also confirmed that USP5 mRNA was highly expressed in pancreatic cancer cell lines compared with normal pancreatic cells. Taken together, we speculated that increased USP5 level is associated with tumor metastasis.

\section{USP5 overexpression promotes the progression and metastasis of pancreatic cancer}

To further evaluate the biological function of USP5 in vitro experiments, we transfected USP5 overexpressing plasmid and observe cell morphology under microscope (Fig. 3A). CCK-8 assay and colony formation experiments showed that overexpression of USP5 in Panc1 cells dramatically increased the proliferation (Fig. 3B-C). Transwell assay and wound healing assay were used to assess the effects of USP5 in metastasis. Transwell assay demonstrated that overexpress USP5 promote both migration and invasion after 24 hours (Fig. 3D-E). Similarly, wound healing assay showed that the healing capability of USP5 overexpression was significantly increased compared with negative control cells after the observation of 24 hours (Fig. 3F). Western blot analysis further affirmed that the expression level of EMT related markers ( $\mathrm{N}$-cadherin, vimentin and $\beta$ catenin) have been changed relatively (Fig. 3G).

\section{USP5 knockdown inhibits the progression and metastasis of pancreatic cancer}

To further identify the role of USP5 in pancreatic cancer, we knockdown the expression of USP5 in AsPC1 cells by shRNA. Cell morphology was observed under microscope (Fig. 4A). The cell viability assay and colony formation assay revealed that cell proliferation was significantly inhibited in USP5 knockdown cells (Fig. 4B-C). In contrast with USP5 overexpression, knockdown USP5 inhibited cell invasion and migration (Fig. 4D-G). Taken together, these results suggested that USP5 could evidently promote pancreatic cancer cells proliferation and metastasis. 
A

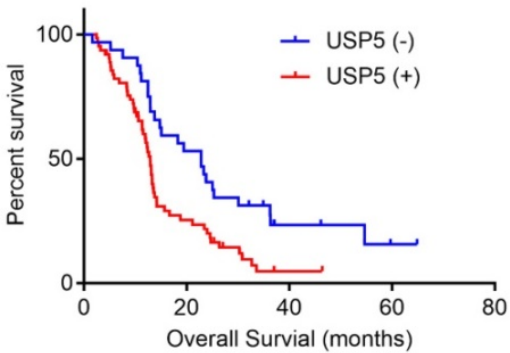

C

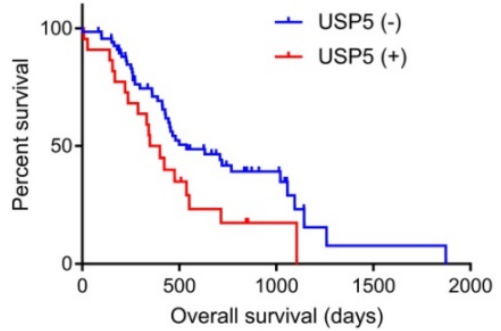

B

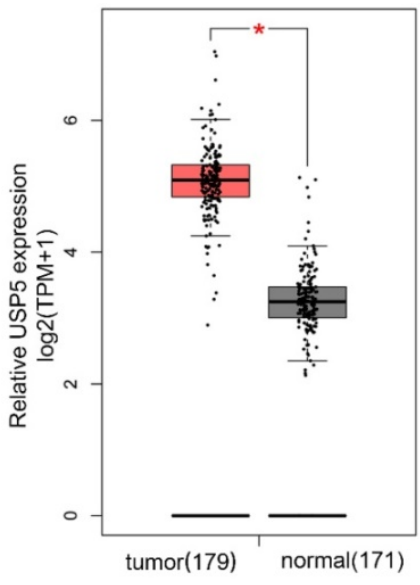

Figure 1. High USP5 expression suggests poor prognosis and shorter OS. (A) The overall survival curves for the high-USP5 expression group and the low-USP5 expression group; the difference is statistically significant $(P<0.05)$. (B) USP5 expression levels in normal or pancreatic cancer tissues retrieved from GEPIA database. TPM, transcripts Per Million. (C) The overall survival curves for USP5 in ICGC database (ICGC-PACA-AU), $P<0.05$.

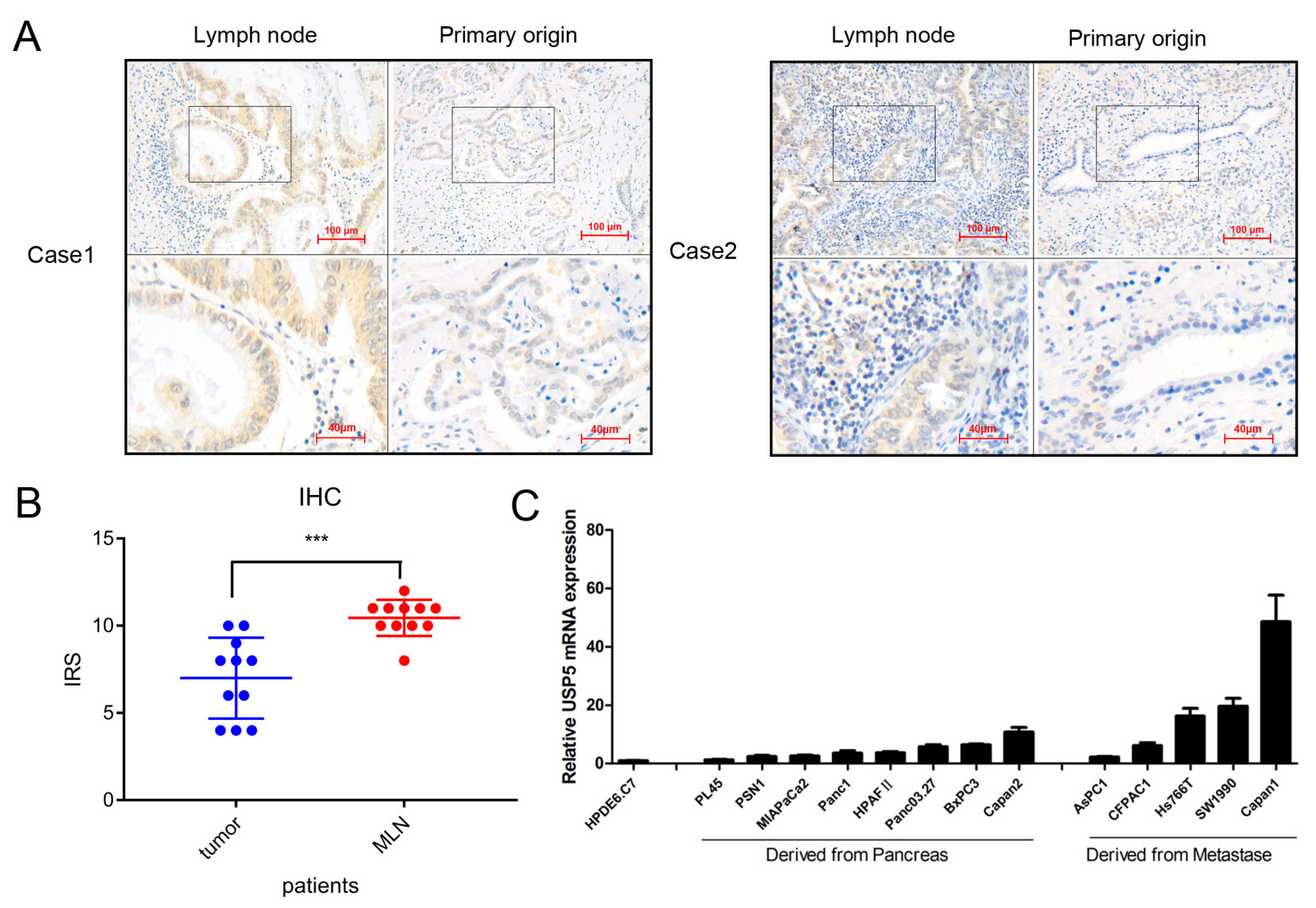

Figure 2. Increased USP5 expression suggests correlation with pancreatic cancer metastasis. (A) USP5 expression in lymph node and primary origin in paraffinembedded tissues from pancreatic cancer patients. (magnification, 200x) (B) IHC score in metastasis lymph node (MLN) and primary origin. (C) The expression of USP5 examined by qPCR in eight cell lines derived from pancreatic cancer, five cell lines derived from metastasis and one normal pancreatic epithelial cell line. $* P<0.05 ; * * P<0.01 ; * * * P<0.001$. 

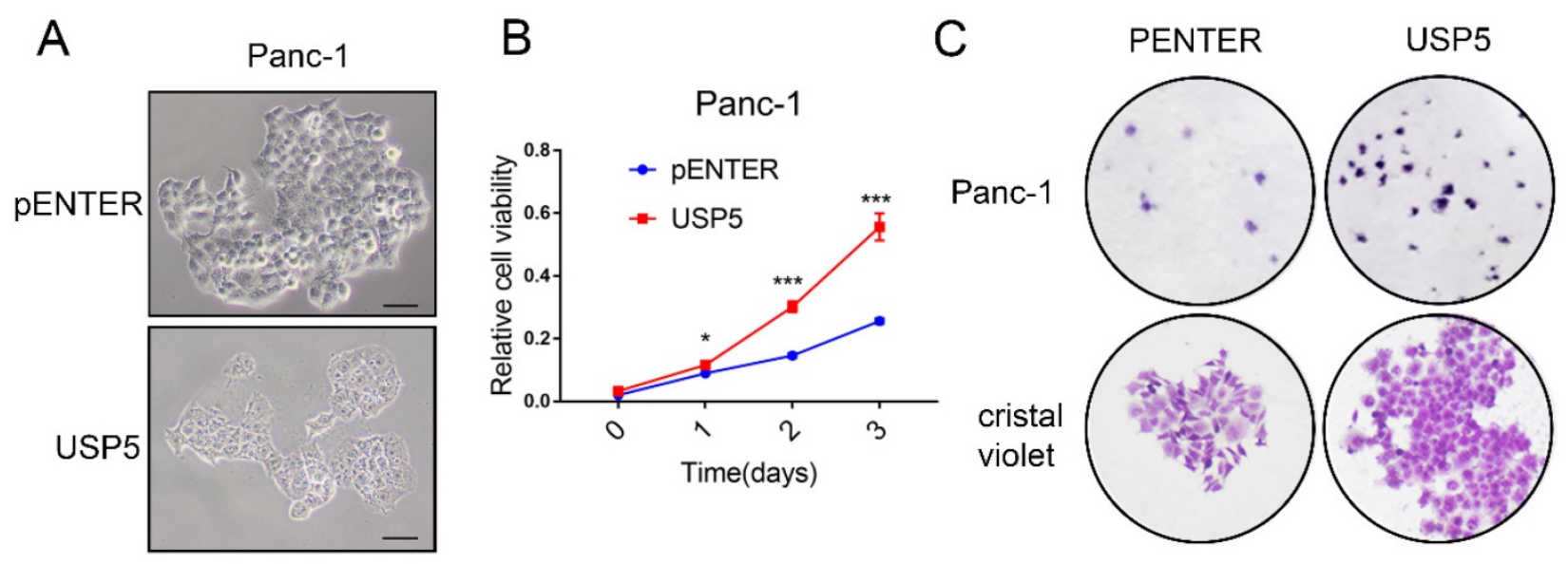

D

Panc-1

$E$
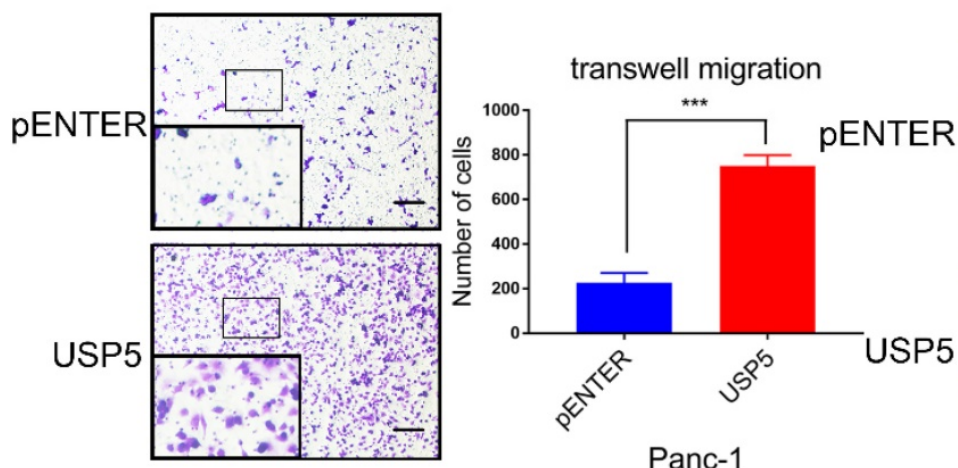

Panc-1

Panc-1
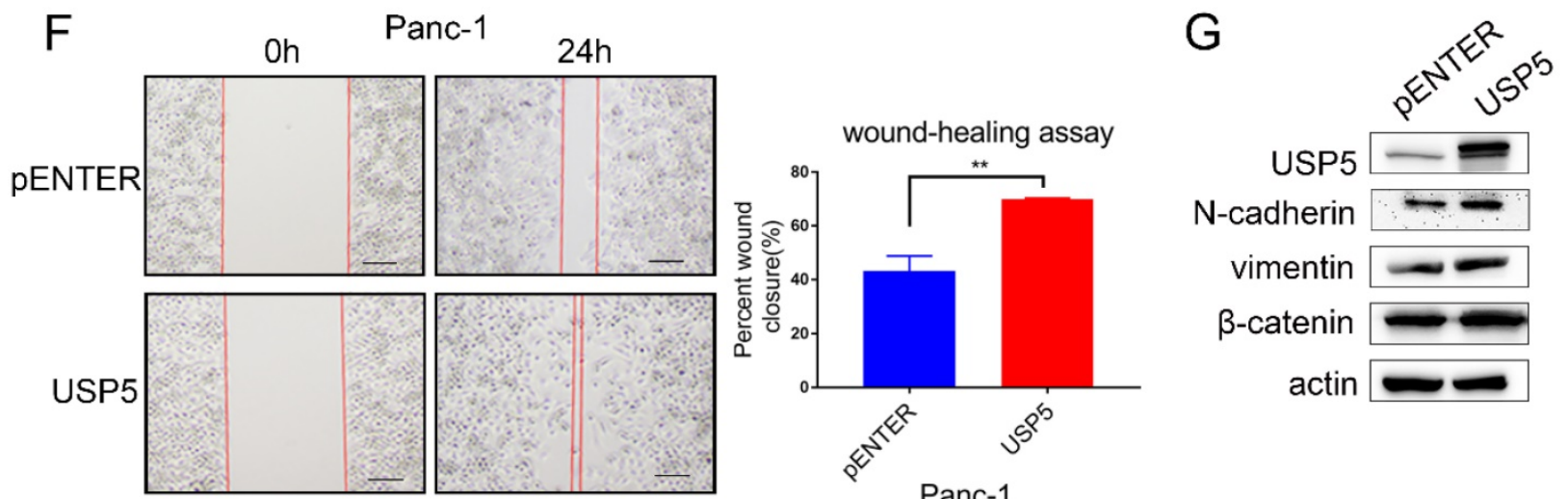

Figure 3. USP5 overexpression promotes the progression and metastasis of pancreatic cancer. (A) Overexpressed USP5 in Pancl cells.(B) Overexpression of USP5 improved the viability of Pancl cells. (C) Overexpression of USP5 enhanced colony formation of the Pancl cells. (D-E) Transwell assays evaluated the effect of USP5 overexpression on migration and invasion of Pancl cells after 24 hours. (F) Wound healing assay of Pancl cells transfected with pENTER and USP5. Results were measured after 24 hours. (G) The expression level of EMT related markers in USP5 overexpression cells. Scale bar $100 \mu \mathrm{m} * P<0.05, * * P<0.01$, $* * * P<0.001$, Student's unpaired t-test.

\section{USP5 mediates STAT3 signaling in pancreatic cancer}

STAT3 is often overexpressed or overactivated in several tumors [12]. A recent study also reported that the ubiquitin ligase Fbw7 induced STAT3 ubiquitination for degradation in diffuse large B-cell lymphoma [13]. However, the effect of USP5 in STAT3 signaling was still unclear. In this study, we found that STAT3 was overactivated, along with the upregulation of USP5 (Fig. 5A). In contrast, knockdown of USP5 suppressed the activation of STAT3 (Fig. 5B).

\section{Discussion}

Ubiquitin-proteasome pathway is a common pathway for protein degradation. Increasing evidence has shown that USP family has important significance 
in tumor cell apoptosis [4], cycle [10], metastasis [6], drug resistance [14] and other biological activities [15, 16]. For instance, USP4 promote tumor progression by regulating $\beta$-Catenin Signaling [17]. It has been proven that USP2 promotes breast cancer metastasis by deubiquitinating MMP2 [18]. Also, USP22 is responsible for hepatocellular carcinoma migration [19]. USP5 has been reported in targeting several proteins, such as P53, FOXM1, SLUG, $\beta$ catenin, c-Maf, TUFM, HDAC2 et al [4-9, 20, 21]. USP5 has also been implicated in a wide variety of cellular events, such as the efficient repair of DNA Double-Strand Breaks, alternative RNA Splicing, neural pain, tumor cell proliferation and progression [6, 9, 15, 16, 22]. Intriguingly, USP5 has been confirmed to mediate p53 signaling [20], wnt- $\beta$-catenin pathway [5], Type I interferon signaling pathway [23], Notch and RTK signaling pathway [24]. A recent study confirmed that upregulated USP5 can promote pancreatic cancer [10]. However, its role in pancreatic cancer still remains further studied.
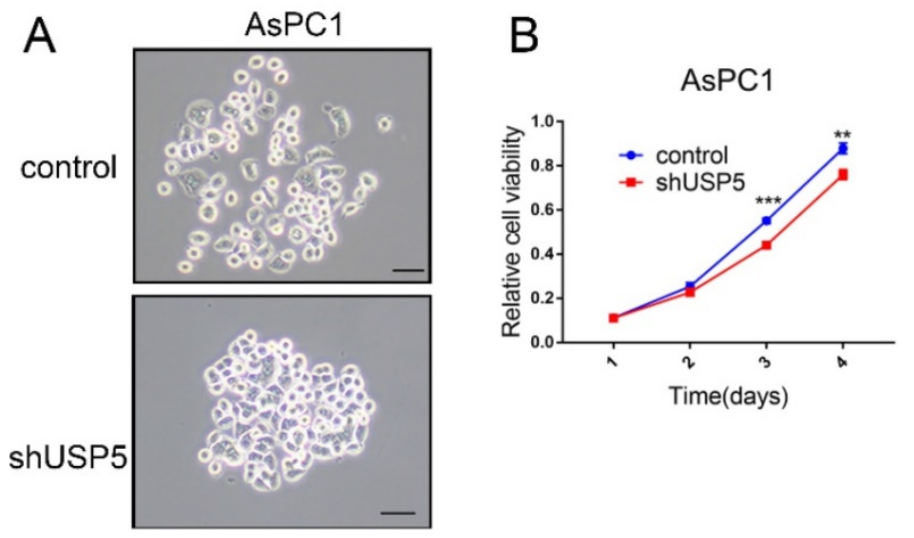

D
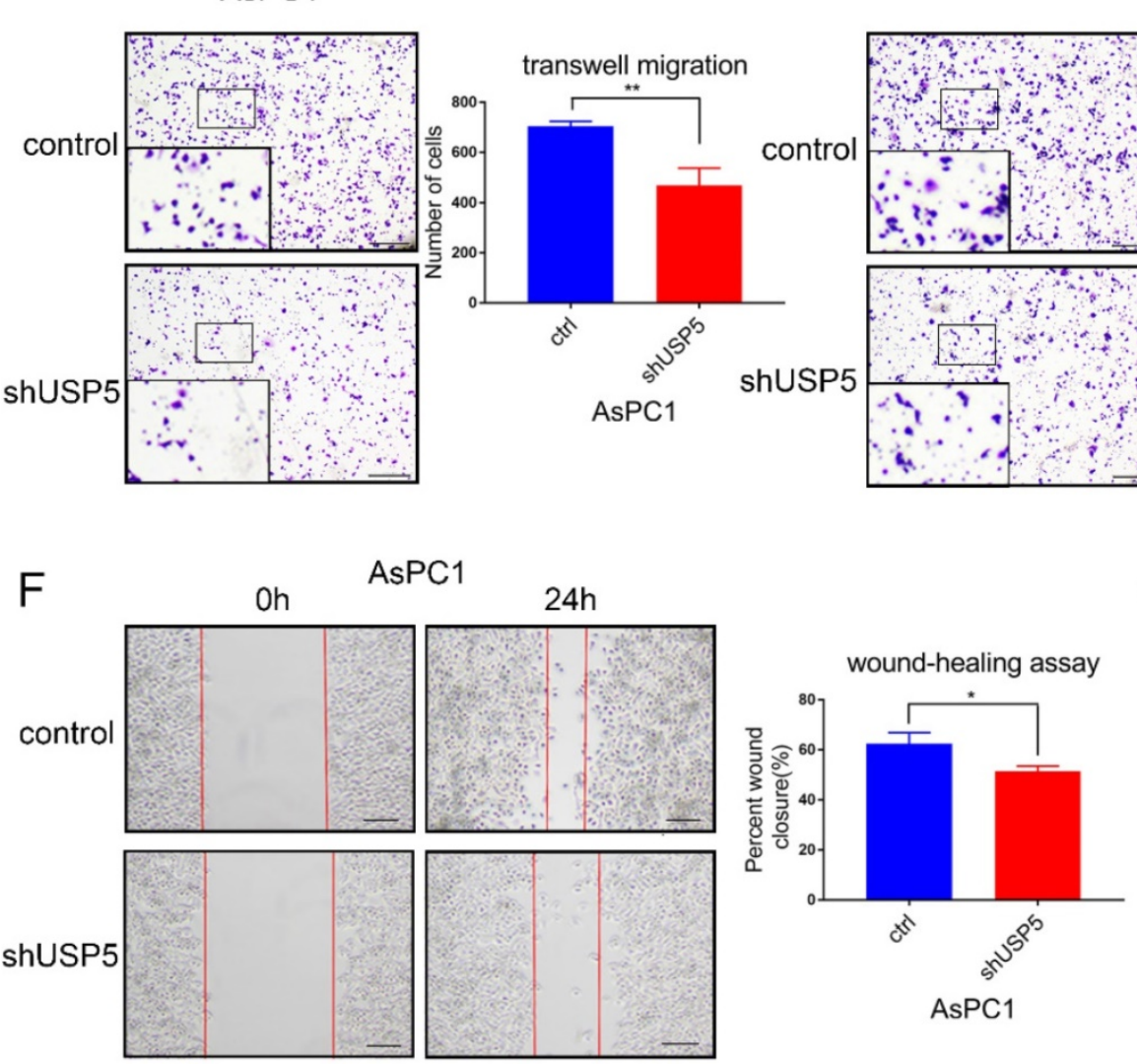

E

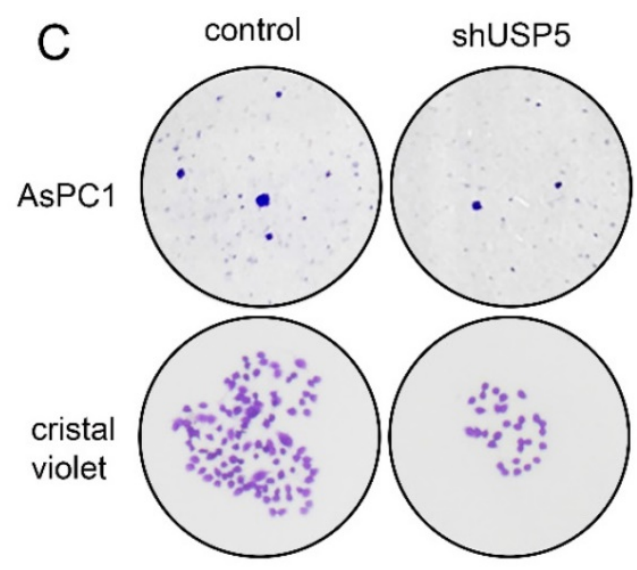

AsPC1

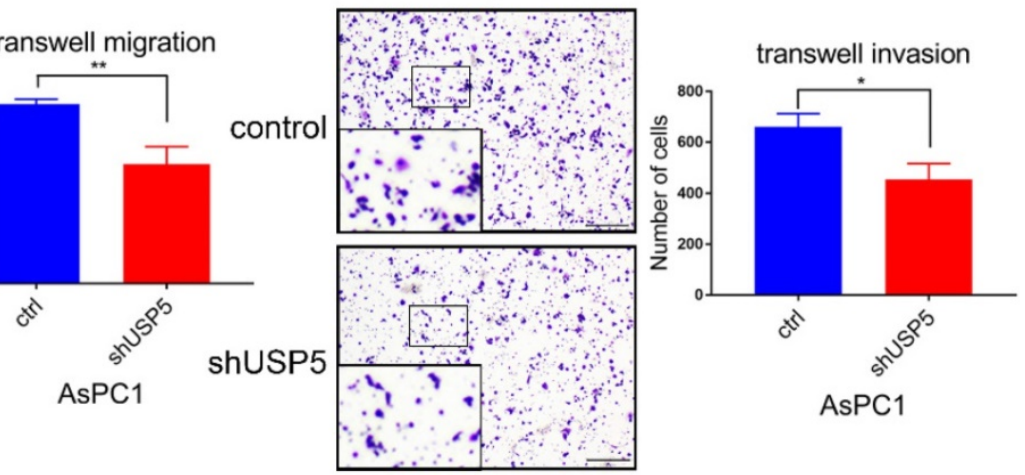

Figure 4. USP5 knockdown inhibits the progression and metastasis of pancreatic cancer. (A) Knockdown USP5 in AsPC1 cells. (B) Knockdown USP5 inhibit the viability of AsPC1 cells.(C) Knockdown USP5 inhibit colony formation of the AsPCl cells. (D-E) Transwell assays evaluated the effect of USP5 knockdown on migration and invasion in AsPCl cells after 24 hours. (F) Wound healing assay of AsPCl cells transfected with shUSP5. Results were measured after 24 hours. (G) The expression level of EMT related markers in USP5 knockdown cells. Scale bar $100 \mu \mathrm{m} * P<0.05$, $* * P<0.01, * * * P<0.001$, Student's unpaired t-test. 

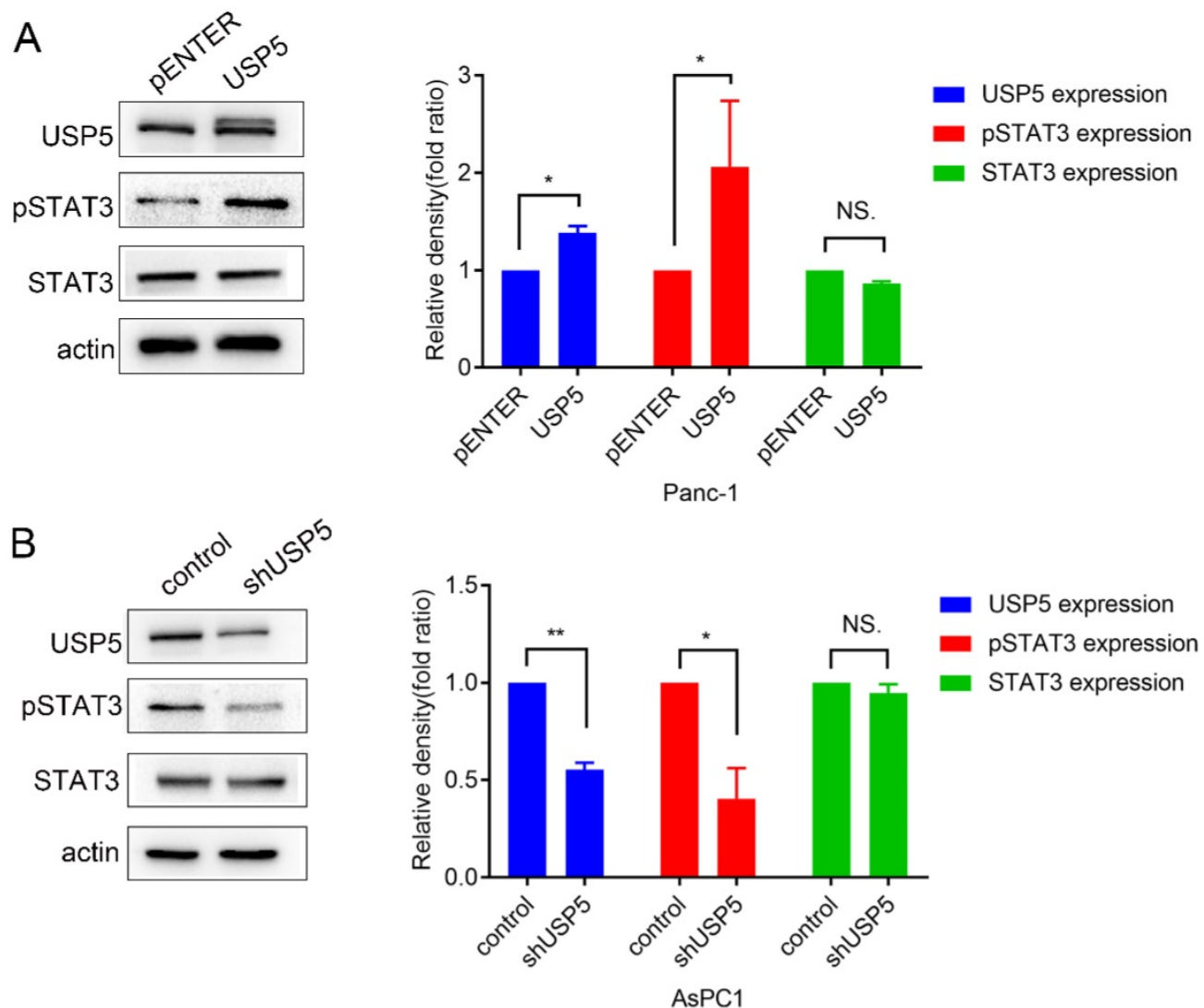

Figure 5. USP5 was proved to mediate STAT3 signaling in pancreatic cancer cells. (A) Overexpress USP5 in Pancl cells activate STAT3 signaling. (B) Knockdown USP5 in AsPC1 cells inhibit STAT3 signaling. Data were quantified by densitometric analysis with Imagel software. $* P<0.05 ; * * P<0.01 ; * * * P<0.001$.

The expression status and clinical significance of USP5 in pancreatic cancer has not been reported extensively. In the current study, we discovered that USP5 expression level is related to tumor differentiation, CEA and CA19-9 levels. Univariate and multivariate analyses demonstrated that high USP5 expression is an independent risk factor for pancreatic cancer. Our results also showed that differentiation is an unfavorable independent risk factor for pancreatic cancer, which have been proved by previous study [25]. In short, patients with high USP5 expression tend to have a poor prognosis, which is consistent with publicly databases.

Recently, there have been several reports on how USP5 promote the growth of cancer. By stabilizing Tu translation elongation factor, USP5 could regulate colorectal cancer cell growth [7]. Moreover, USP5 stabilized FoxM1 protein in pancreatic ductal adenocarcinoma and regulated cell proliferation via FoxM1 proteins [9]. This has been confirmed in our experiments.

In previous study, USP5 was found promoting EMT by stabilizing SLUG in hepatocellular carcinoma [6]. In our study, we reported that the expression of USP5 is higher in metastasis than in primary tumor. This was confirmed in clinical patient samples and pancreatic cancer cell lines, respectively. To further investigate how USP5 affect pancreatic cancer migration and invasion, we overexpressed USP5 in Panc1 cells and knockdown USP5 in AsPC1 cells. Results elucidated that USP5 contribute to cell invasion and metastasis in pancreatic cancer.

The effect of USP5 in progression and migration in pancreatic cancer has been confirmed, but its mechanism was still unknown. In this study, we confirmed that USP5 mediated STAT3 signaling. The signal transducer and activator of transcription 3 (STAT3) is an important signaling mediator, which plays significant roles in a vast array of biological processes [26-28]. It has been confirmed that overexpression or overactivation of STAT3 is required for tumorigenesis and metastasis. Thus, USP5 may promote pancreatic cancer progression and metastasis through enhancing STAT3 signaling.

Small molecules inhibitor WP1130 [29] and Chinese medicine Formononetin [6] were reported to inhibit USP5, which further indicated that targeting USP5 might be an effective strategy in pancreatic cancer treatment.

In conclusion, our study identifies that USP5 is highly expressed in pancreatic cancer and might have clinical significance for patients with pancreatic 
cancer. USP5 overexpression is critical in both pancreatic cancer progression and metastasis via enhancing STAT3 signaling. Therefore, USP5 might be a potential novel target gene in pancreatic cancer.

\section{Abbreviations}

USP5: Ubiquitin specific peptidase 5; IHC: immunohistochemistry; DUBs: Deubiquitinating enzymes; USPs: Ubiquitin-specific proteases; STAT3: signal transducer and activator of transcription 3; qPCR: Quantitative real-time PCR; MLN: metastasis lymph node.

\section{Acknowledgements}

\section{Funding}

This study is funded by following foundations:

- Harbin Medical University Research and Practice Innovation Project (No. YJSKYCX201960HYD);

- National Natural Science Foundation of China (No. 81672428, 81872427);

- Applied technology research and development program of Heilongjiang Province (No. GA19C002);

- Haiyan Research Fund (No. JJQN2020-07);

- Nn10 Excellent Discipline Construction Program (No. Hepatobiliary and Pancreatic Tumor 2017);

- Central guides' local science and technology development project (No. ZY16A07).

\section{Authors' contribution statements}

Jie Lian performed the experiments, analyzed the data, and wrote the manuscript. Chao Liu helped conceived and designed the experiments. Xin Guan and Bojun Wang participated in part of experiments. Yuanfei Yao revised the manuscript. Dan Su, Yue Ma and Lin Fang collected clinical samples. Yanqiao Zhang conceived and designed the study, participated in its co-ordination and wrote the manuscript. All authors read and approved the final manuscript.

\section{Ethics committee approval and patient consent}

For the use of these clinical materials for research purposes, prior patient consent and approval was obtained from the Institutional Research Ethics Committee of Harbin Medical University Cancer Hospital. The number of ethical approvals is KY2018-12.

\section{Competing Interests}

The authors have declared that no competing interest exists.

\section{References}

1. Siegel RL, Miller KD, Jemal A. Cancer statistics, 2019. CA: a cancer journal for clinicians. 2019; 69: 7-34.

2. Kamisawa T, Wood LD, Itoi T, Takaori K. Pancreatic cancer. Lancet (London, England). 2016; 388: 73-85.

3. Nijman SM, Luna-Vargas MP, Velds A, Brummelkamp TR, Dirac AM, Sixma TK, et al. A genomic and functional inventory of deubiquitinating enzymes. Cell. 2005; 123: 773-86.

4. Wang S, Juan J, Zhang Z, Du Y, Xu Y, Tong J, et al. Inhibition of the deubiquitinase USP5 leads to c-Maf protein degradation and myeloma cell apoptosis. Cell death \& disease. 2017; 8: e3058.

5. Ma X, Qi W, Pan H, Yang F, Deng J. Overexpression of USP5 contributes to tumorigenesis in non-small cell lung cancer via the stabilization of $\beta$-catenin protein. American journal of cancer research. 2018; 8: 2284-95.

6. Meng J, Ai X, Lei Y, Zhong W, Qian B, Qiao K, et al. USP5 promotes epithelial-mesenchymal transition by stabilizing SLUG in hepatocellular carcinoma. Theranostics. 2019; 9: 573-87.

7. Xu X, Huang A, Cui X, Han K, Hou X, Wang Q, et al. Ubiquitin specific peptidase 5 regulates colorectal cancer cell growth by stabilizing Tu translation elongation factor. Theranostics. 2019; 9: 4208-20.

8. Du Y, Lin J, Zhang R, Yang W, Quan $\mathrm{H}$, Zang L, et al. Ubiquitin specific peptidase 5 promotes ovarian cancer cell proliferation through deubiquitinating HDAC2. Aging. 2019; 11: 9778-93.

9. Li XY, Wu HY, Mao XF, Jiang LX, Wang YX. USP5 promotes tumorigenesis and progression of pancreatic cancer by stabilizing FoxM1 protein. Biochemical and biophysical research communications. 2017; 492: 48-54.

10. Kaistha BP, Krattenmacher A, Fredebohm J, Schmidt H, Behrens D, Widder M, et al. The deubiquitinating enzyme USP5 promotes pancreatic cancer via modulating cell cycle regulators. Oncotarget. 2017; 8: 66215-25.

11. Liu C, Shi J, Li Q, Li Z, Lou C, Zhao Q, et al. STAT1-mediated inhibition of FOXM1 enhances gemcitabine sensitivity in pancreatic cancer. Clinical science (London, England : 1979). 2019; 133: 645-63.

12. Peyser ND, Freilino M, Wang L, Zeng Y, Li H, Johnson DE, et al. Frequent promoter hypermethylation of PTPRT increases STAT3 activation and sensitivity to STAT3 inhibition in head and neck cancer. Oncogene. 2016; 35 : 1163-9.

13. Yao S, Xu F, Chen Y, Ge Y, Zhang F, Huang H, et al. Fbw7 regulates apoptosis in activated B-cell like diffuse large B-cell lymphoma by targeting Stat3 for ubiquitylation and degradation. Journal of experimental \& clinical cancer research : CR. 2017; 36: 10.

14. Zhang Z, Gao W, Zhou L, Chen Y, Qin S, Zhang L, et al. Repurposing Brigatinib for the Treatment of Colorectal Cancer Based on Inhibition of ER-phagy. Theranostics. 2019; 9: 4878-92.

15. Gadotti VM, Caballero AG, Berger ND, Gladding CM, Chen L, Pfeifer TA, et al. Small organic molecule disruptors of Cav3.2 - USP5 interactions reverse inflammatory and neuropathic pain. Molecular pain. 2015; 11: 12.

16. Izaguirre DI, Zhu W, Hai T, Cheung HC, Krahe R, Cote GJ. PTBP1-dependent regulation of USP5 alternative RNA splicing plays a role in glioblastoma tumorigenesis. Molecular carcinogenesis. 2012; 51: 895-906.

17. Nguyen HH, Kim T, Nguyen T, Hahn MJ, Yun SI, Kim KK. A Selective Inhibitor of Ubiquitin-Specific Protease 4 Suppresses Colorectal Cancer Progression by Regulating $\beta$-Catenin Signaling. Cellular physiology and biochemistry : international journal of experimental cellular physiology, biochemistry, and pharmacology. 2019; 53: 157-71.

18. Qu Q, Mao Y, Xiao G, Fei X, Wang J, Zhang Y, et al. USP2 promotes cell migration and invasion in triple negative breast cancer cell lines. Tumour biology : the journal of the International Society for Oncodevelopmental Biology and Medicine. 2015; 36: 5415-23.

19. Ling S, Li J, Shan Q, Dai H, Lu D, Wen X, et al. USP22 mediates the multidrug resistance of hepatocellular carcinoma via the SIRT1/AKT/MRP1 signaling pathway. Molecular oncology. 2017; 11: 682-95.

20. Liu Y, Wang WM, Zou LY, Li L, Feng L, Pan MZ, et al. Ubiquitin specific peptidase 5 mediates Histidine-rich protein Hpn induced cell apoptosis in hepatocellular carcinoma through P14-P53 signaling. Proteomics. 2017; 17: 1600350.

21. Potu H, Peterson LF, Pal A, Verhaegen M, Cao J, Talpaz M, et al. Usp5 links suppression of p53 and FAS levels in melanoma to the BRAF pathway. Oncotarget. 2014; 5: 5559-69.

22. Nakajima S, Lan L, Wei L, Hsieh CL, Rapić-Otrin V, Yasui A, et al. Ubiquitin-specific protease 5 is required for the efficient repair of DNA double-strand breaks. PloS one. 2014; 9: e84899.

23. Liu $\mathrm{Q}, \mathrm{Wu} \mathrm{Y}, \mathrm{Qin} \mathrm{Y}, \mathrm{Hu} \mathrm{J}, \mathrm{Xie} \mathrm{W}$, Qin FX, et al. Broad and diverse mechanisms used by deubiquitinase family members in regulating the type I interferon signaling pathway during antiviral responses. Science advances. 2018; 4: eaar2824.

24. Ling $X$, Huang $\mathrm{O}, \mathrm{Xu} Y$, Jin $Y$, Feng $Y$, Shi $\mathrm{W}$, et al. The deubiquitinating enzyme Usp5 regulates Notch and RTK signaling during Drosophila eye development. FEBS letters. 2017; 591: 875-88.

25. Kardosh A, Lichtensztajn DY, Gubens MA, Kunz PL, Fisher GA, Clarke CA. Long-Term Survivors of Pancreatic Cancer: A California Population-Based Study. Pancreas. 2018; 47: 958-66. 
26. Kitamura H, Ohno Y, Toyoshima Y, Ohtake J, Homma S, Kawamura H, et al. Interleukin-6/STAT3 signaling as a promising target to improve the efficacy of cancer immunotherapy. Cancer science. 2017; 108: 1947-52.

27. Zhu F, Wang KB, Rui L. STAT3 Activation and Oncogenesis in Lymphoma. Cancers. 2019; 12: 19

28. Li Z, Qin X, Bian W, Li Y, Shan B, Yao Z, et al. Exosomal lncRNA ZFAS1 regulates esophageal squamous cell carcinoma cell proliferation, invasion, migration and apoptosis via microRNA-124/STAT3 axis. Journal of experimental \& clinical cancer research : CR. 2019; 38: 477.

29. Kapuria V, Peterson LF, Fang D, Bornmann WG, Talpaz M, Donato NJ.

Deubiquitinase inhibition by small-molecule WP1130 triggers aggresome formation and tumor cell apoptosis. Cancer research. 2010; 70: 9265-76. 